\title{
Particular aspects of calculating affecting factors of fireballs emerging from aircraft crash.
}

\author{
Alexander Komarov and Nikolay Gromov* \\ Moscow State University of Civil Engineering, Yaroslavskoe shosse, 26, Moscow, 129337, Russia
}

\begin{abstract}
The article reveals an algorithm of calculating the heat loads which emerge during fireball-involving accidents. By way of illustration it considers a situation when a heavy aircraft crashes in the vicinity of a typical reactor building of a nuclear power plant. The given algorithm provides a quite detailed and accurate calculation of maximum heat load values that accompany the accident.
\end{abstract}

\section{Introduction}

When considering safety issues at nuclear power plants (NPP), it is required to determine a potential threat that an aircraft crash in the vicinity of the plant might impose. In this respect, there normally assumed the most conservative accident scenarios. Thus, when dealing with a scenario involving an aircraft crash in the vicinity of an NPP, in most cases a wide-body airplane like Boeing-747 is considered.

It appears somewhat problematic to nominally apply governing documents regulating calculation of fireball parameters to this very accidental situation, therefore, the present article brings in the basic principles and some research findings aiming to solve the problem. A Boeing-747 will be considered as a potentially hazardous source.

\section{Methods}

Let's look at the physical processes which occur in case of an aircraft crash and which entail a generation of a fireball.

The aircraft crash causes a jet fuel (kerosene) spillage and spray dissemination resulting in a formation of a cloud of dispersed drops. The energy of heated engine parts and kinetic drop energy provides creation of fuel vapors and dispersed kerosene drops. When the cloud ignites, there appears a fireball. The mixture of vapors and fuel drops burns off the ball's surface as soon as oxygen gets in. This generates a significant amount of heat, while the fireball 'surfaces' being driven by the buoyancy force.

${ }^{*}$ Corresponding author: newdayru@bk.ru 
An area of rich vapor-air mixture at the moment of aircraft crash may emerge only due to fuel evaporation from hot engine parts. The engine is capable of evaporating a significant amount of liquid fuel that comes in contact with it during the accident.

Following consultations with aviation engine experts, it was found that the temperature of any aircraft engine may on the average be estimated as $460^{\circ} \mathrm{C}$.

The Boeing-747's engine weighs 4300kg. The overall heat energy of one engine, which can be spent for fuel evaporation, will comprise $Q=0,81 \cdot 10^{6} \mathrm{~kJ}$. This will allow to evaporate no more than $Z=Q /\left(Q_{F}+Q_{H}\right)=1520 \mathrm{~kg}$ of fuel, where $Q_{F}=283 \mathrm{~kJ} / \mathrm{kg}$ is fuel evaporation heat, $Q_{H}=250 \mathrm{~kJ} / \mathrm{kg}$ is heat required to warm the fuel up to the boiling temperature. The assumed emergency aircraft has four engines. So in case of a crash event, the maximum fuel quantity, which will be able to participate in vapor-air cloud formation, will not exceed $M_{T}=6080 \mathrm{~kg}$. Then, the maximum radius of the fireball generated by one engine will not exceed $R_{S}=20 \mathrm{~m}$.

The calculation of the fireball's behavior (the 'surfacing'/ascending process) as well as the calculation of sedimentation and 'surfacing' of heavy and light vapor-air clouds correspondingly were conducted numerically based on the well-known Godunov's method described in [1]. The numerical scheme of the Godunov's method utilizes the arbitrary discontinuity decay theory $[1,2]$. The basics of the discontinuity decay phenomenon can be found in any major continuum mechanics course (for example, $[3,4,5,6]$ ).

The problem of the arbitrary discontinuity decay with the reference to the numerical scheme is taken down to the simultaneous non-linear equations in pressure $P_{p}$ and speed $U_{p}$ of the medium at the discontinuity decay point. These value determine the flow of mass $M_{i, i+1}$ and impulse $I_{i, i+1}$, which are being swapped between nearby computational cells $i$ and $i+1$.

The calculation of changes in medium concentration or density inside the cell (depending on the considered problem) is performed according to the diffusive behavior pattern of continuous medium.

In order to verify the validity of the math model and feasibility of the program code applied for calculation of the dynamic processes accompanying explosive events, we used the experiment results that were given in $[7,8,9]$.

Photos in Figure 1 demonstrate 'surfacing' of deflagration explosion products. The time step constituted about 40ms. [7,8,9]. There were conducted calculations for initial conditions assumed in the experiment as well as for the moments in time that were registered in the photos.

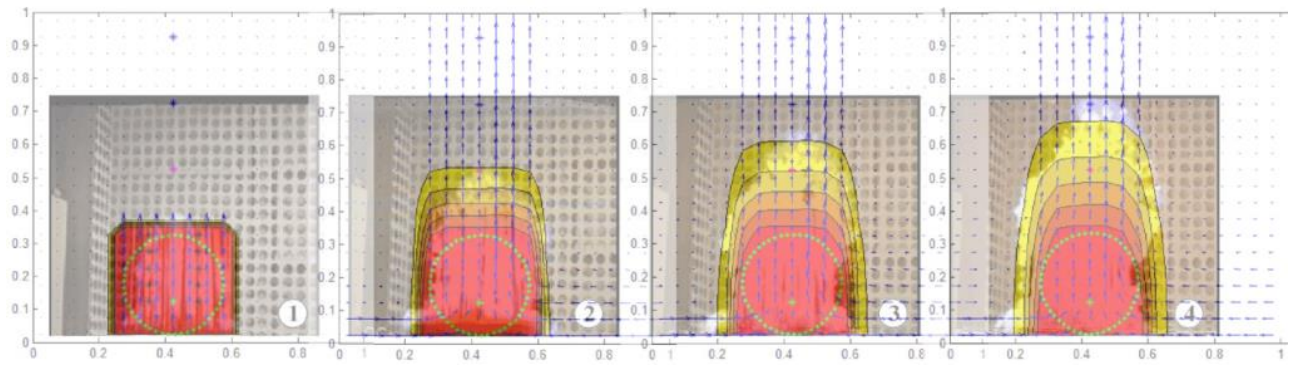

Fig. 1. Photos of 'surfacing' of combustion products and overlaid calculated positions of explosion products. 
In order to make a vivid comparison of calculation results acquired through the numerical scheme in use with those obtained during experiments, the calculations are overlaid on the experiment photos in Figure 1. Besides, Figure 1 demonstrates the calculated velocity vectors of the flows that accompany the 'surfacing' process of combustion products. data.

The given figure suggests that the calculation results are quite close to the experimental

For calculations of possible heat loads emerging from an aircraft crash on the NPP site we will use a well-known ratio [10.11] describing the slope rate of diffusive radiation transfer from a sphere with radius $R_{S}$ onto a surface element: $F_{q}=\frac{\cos \varphi}{R^{2}} \cdot R_{S}{ }^{2}$, where $\varphi$ is the angle between the normal to the surface element and the vector connecting the ball's and element's centers.

Let's take a look at calculation results of the 'surfacing' process of an emerged burning cloud (fireball). It is assumed that the mixture burns quite intensively which causes fast emission of combustion products, a significant increase of the upward buoyancy force, a high climb rate of the ball and an increase in density of heat radiation.

Figure 2 provides instant locations of the formed fireball for several moments in time (the time step constituted 1 second).

Figure 2 provides several moments of the flame propagation process registered during Experiment I.
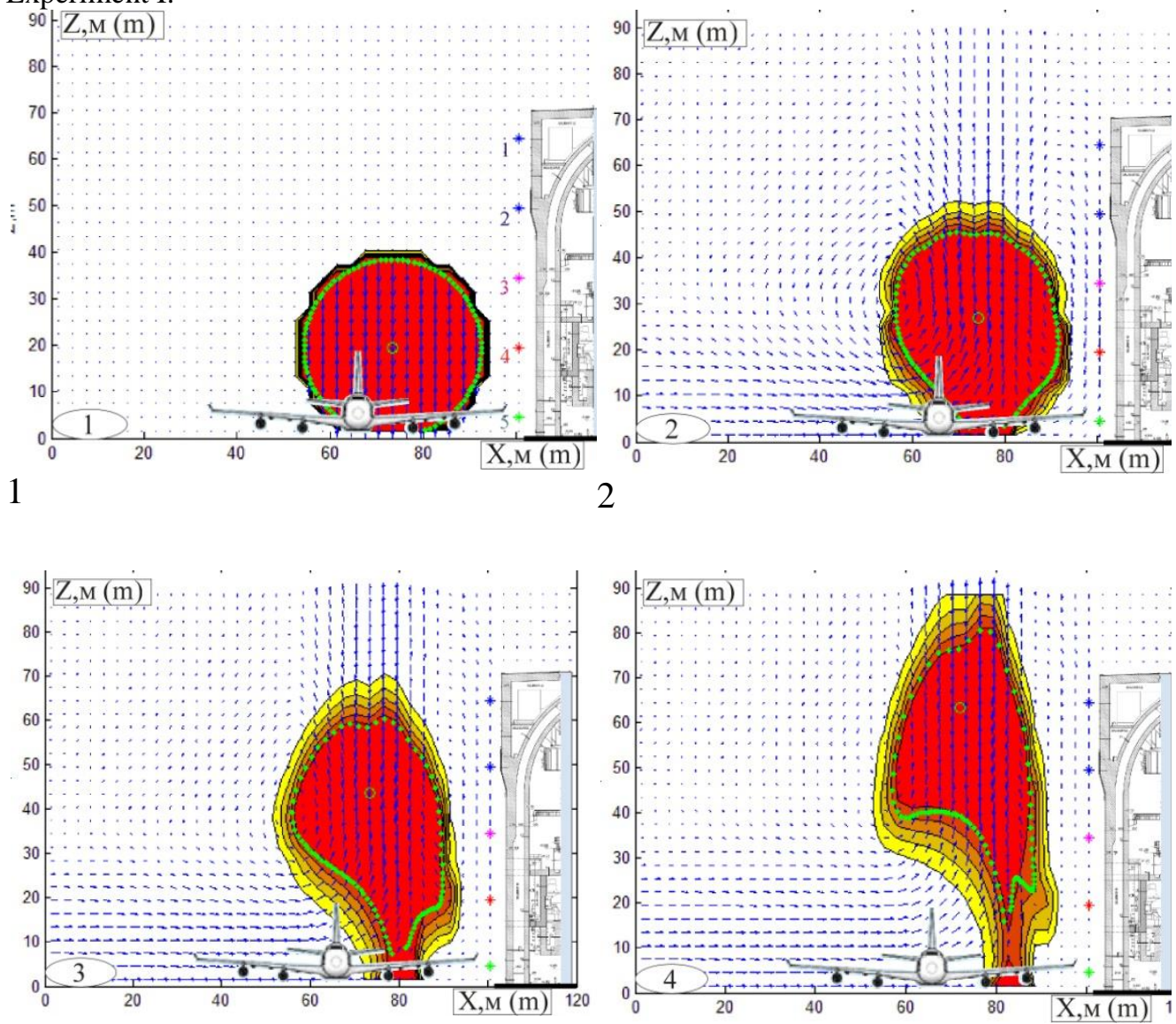
Fig. 2. Fireball locations for several moments in time.

1 - starting position of the fireball (the ignition moment);

2 - cloud position in 1second after ignition; 3 - in 2 seconds; 4 - in 3 seconds.

The given pictures show velocity vectors of the air flow which accompanies the rising fireball. The calculation was conducted in boundary conditions, which assume that there is an NPP reactor building 30m away from the center of the fireball. The condition of fluid impermeability was assumed at the border of building. In order to get a general idea, Figure 2 provides a scaled scheme of a Boeing-747 aircraft. Later there will be provided heat flow parameters for the five points marked in the figures left of the reactor building.

While 'surfacing', the combustion products that emit during the fireball's burning, mix with the air. The plots demonstrate isolines of equal volume concentrations of the combustion products (see Figure 2). The outer isoline reflects a 10\% content of explosion products in the mixture surrounding the fireball. That means that in those places hot explosive products get diluted by the ambient air. Other isolines demonstrate $20 \%, 30 \%$, $40 \%$ and $50 \%$. Further away, there is basically only combustion products or 'fresh' mixture.

Figure 3 provides time laws of heat radiation intensity present at the five points of the reactor building. The average flow of heat radiation implies the value of $E=\left(E_{X}+E_{Z}\right) / 2$, where $E_{X}$ is the heat flow affecting vertical surfaces, whereas $E_{Z}$ is the heat flow affecting horizontal surfaces.
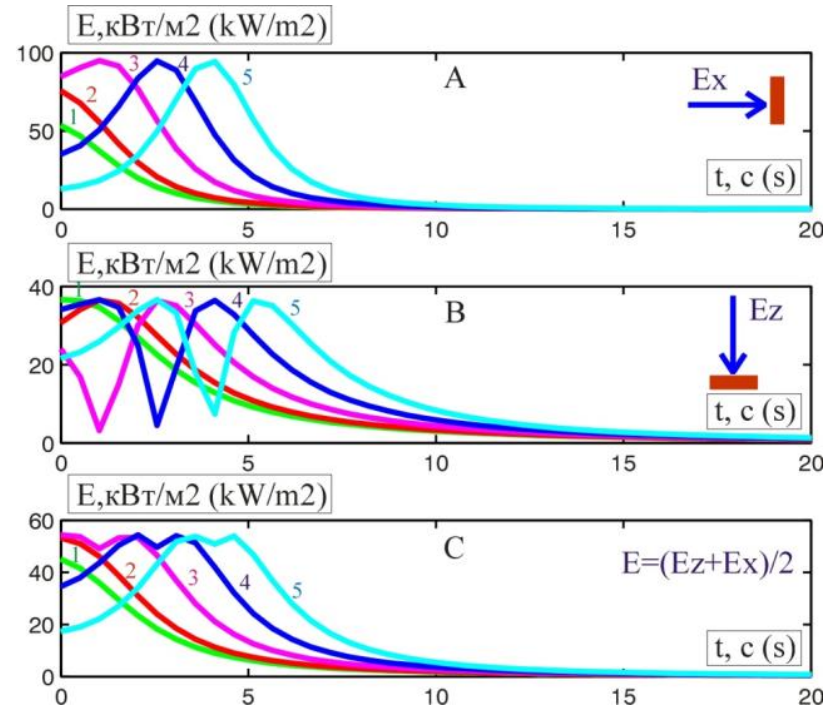

Fig. 3. Time laws of heat radiation intensity present at the five points of the reactor building, (the locations of the points on the front-end wall of the building are indicated in Figure 2): A - intensity of radiation affecting vertical surfaces; B - intensity of radiation affecting horizontal surfaces; C average radiation intensity.

The given figure suggests that the maximum heat radiation intensity from a fireball does not exceed $95 \mathrm{~kW} / \mathrm{m}^{2}$. The maximum heat loads affect the building parts which are vertically oriented. The duration of exposure does not exceed 3 seconds.

Figure 4 provides isolines of equal radiation doses that will affect the vertically-oriented surfaces. It's worth reminding that the heat radiation dose $Q$ is the integral of heat radiation 
intensity with respect to time - $Q=\int E d t$ and is expressed in units of $\mathrm{kJ} / \mathrm{m}^{2}$. The calculations imply the following levels of equal heat radiation doses from the fireball: $Q=$ $60,120,220,320,400,500$ and $600 \mathrm{~kJ} / \mathrm{m}^{2}$. Dose levels: 120,220 and $320 \mathrm{~kJ} / \mathrm{m}^{2}$ correspond to the critical values of heat radiation. A dose level of $120 \mathrm{~kJ} / \mathrm{m}^{2}$ causes a first-degree burn; a dose of $220 \mathrm{~kJ} / \mathrm{m}^{2}$ causes a second-degree burn; a dose of $320 \mathrm{~kJ} / \mathrm{m}^{2}$ corresponds to a third-degree burn. The given figure contains scaled pictures of an aircraft, the reactor building and the initial position of the fireball.

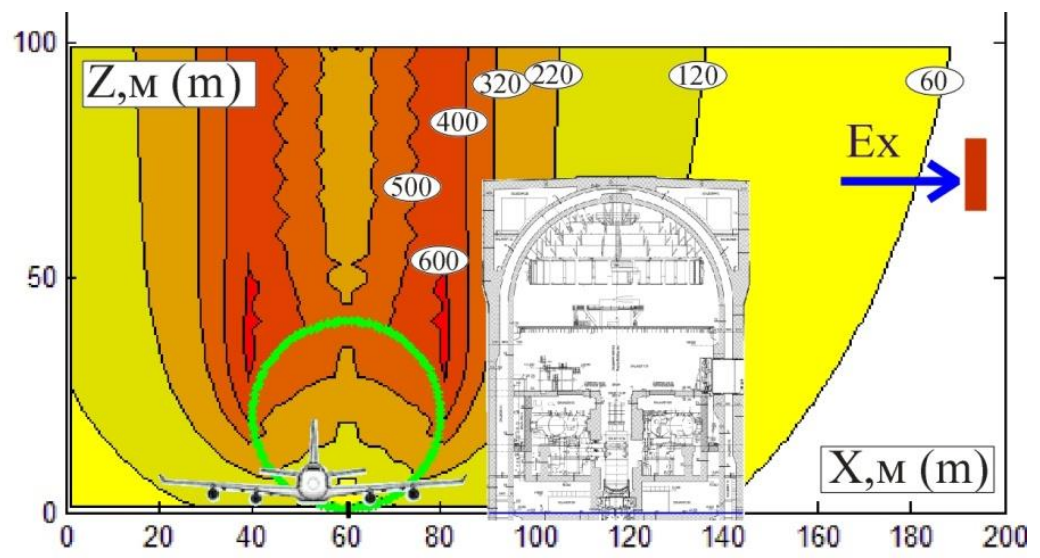

Fig. 4. Isolines of equal radiation doses from a fireball that will affect vertical surfaces in the course of the second accidental scenario. Heat radiation flow $\mathrm{E}_{\mathrm{X}}$.

More detailed data about the heat radiation doses which will affect vertically-oriented parts of the front-end wall of the NPP reactor building are given in Figure 5. The OX-axis of the plot in Figure 5 contains critical values of heat radiation doses: 120, 220 and 320 $\mathrm{kJ} / \mathrm{m}^{2}$. Calculations have shown that if the fireball burns out and, thus, rises rapidly, the maximum heat radiation dose affecting the front-end wall of the reactor building does not exceed $355 \mathrm{~kJ} / \mathrm{m}^{2}$.

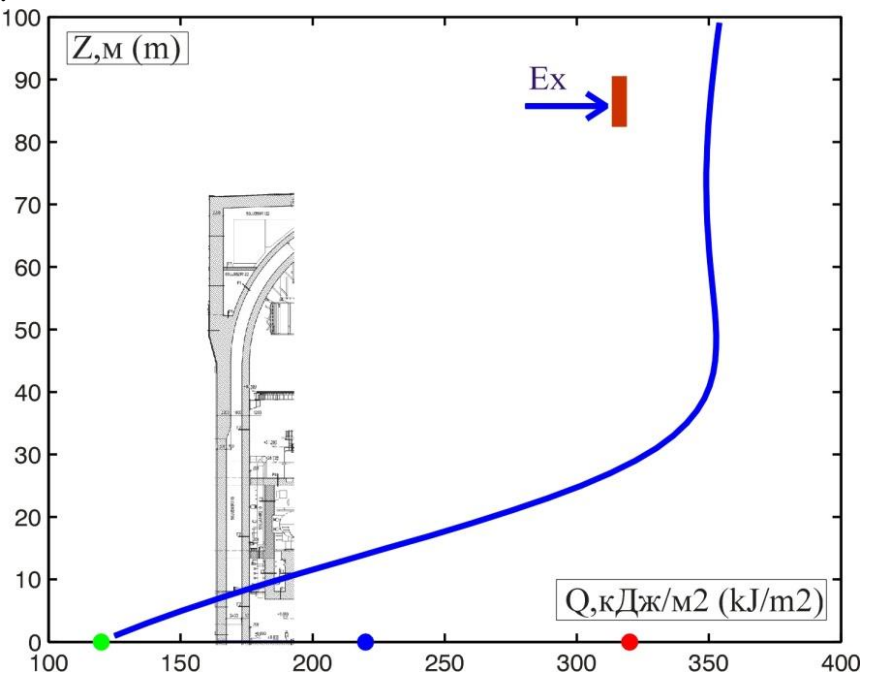

Fig. 5. The distribution of radiation doses from the fireball over vertical surfaces of the front-end wall of the reactor building. Heat radiation flow $\mathrm{E}_{\mathrm{X}}$. 
The demonstrated algorithm allows to acquire all necessary information about heat loads generated by a fireball in case of an aircraft crash. The algorithm can be used to calculate parameters of affecting factors for any accident involving a fireball.

\section{Conclusions}

The article provides a general algorithm of calculating the heat loads which emerge during fireball-involving accidents.

As an example, the algorithm is used for a scenario that includes a crash of a wide-body commercial aircraft in the vicinity of a typical reactor building of an NPP. The calculations assume the worst-case scenario of an aircraft crash in the vicinity of the building and determine the maximum parameters of heat loads: spacial distribution of maximum heat radiation doses affecting the front-end wall of the building; time laws of heat radiation intensity from the fireball in any location including the front-end wall; duration of heat radiation exposure in any location, etc.

The demonstrated algorithm allows to acquire all necessary information about heat loads generated by a fireball in case of an aircraft crash.

\section{References}

1. Numerical Calculation of Multidimensional Problems of Gas Dynamics Edited by S.K. Godunov M. Nauka. 1976. P.400.

2. S.K. Goduniv, V.S. Ryabenkiy Difference schemes. M. Nauka. 1973. P.400.

3. L.D. Landau, U.M. Lifshits Hydrodynamics. M. Nauka. 1988. P.733.

4. L.D. Landau, U.M. Lifshits Continuum mechanics M. Technical and Theoretical Literature Publishing 1953. P.788.

5. L.G. Loitsyansky Fluid and gas mechanics. M. Nauka. 1973. P.847.

6. N.E. Kochin, I.A. Kibel, N.V. Rose Theoretical hydromechanics, vol. 1-2. Technical and Theoretical Literature Publishing M. 1955.

7. A.A. Komarov Forecasting Loads and Estimating Consequences of Their Impact on Buildings and Structures. Doctoral dissertation in 05.26.03 - Fire safety and HSE (construction building). M. MSCU. 2001. - 460p.

8. A.A. Abrosimov, A.A. Komarov The Mechanism of Explosive Loads At Oil Refinery Plants. The Oil, Gas and Business Mechanism. 2002 Issue 6 (50). p.58-61.

9. A.A. Komarov Calculation of Gas-Dynamic Characteristics of Flows at Deflagration Explosions at Outdoor Installations. The Fire and Explosion Safety Magazine, 2002, Vol.11. No.5. p.15-18.

10. Yu.A. Koshmarov, M.P. Bashkirtsev. Thermodynamics and heat transfer in firefighting. M: Higher Engineering and Firefighting Technical School, Ministry of Internal Affairs of the USSR, 1987. - $440 \mathrm{p}$.

11. M.P. Bashkirtsev. Basics of fire thermal physics. M.: Stroyizdat, 1978. - 198 p. 